\title{
Correction to: Usefulness of Genetic Testing in Hypertrophic Cardiomyopathy: an Analysis Using Real-World Data
}

\author{
M. Alejandra Restrepo-Cordoba ${ }^{1}$ - Oscar Campuzano ${ }^{2,3,4}$ - Tomás Ripoll-Vera ${ }^{5}$ - Marta Cobo-Marcos ${ }^{1,4}$. \\ Irene Mademont-Soler ${ }^{2,4}$ • José M. Gámez ${ }^{5}$. Fernando Dominguez ${ }^{1,4,6}$ - Esther Gonzalez-Lopez ${ }^{1,4,5}$. \\ Laura Padron-Barthe ${ }^{1,4,6}$ • Enrique Lara-Pezzi ${ }^{4,6}$ - Luis Alonso-Pulpon ${ }^{1,4}$ - Ramon Brugada ${ }^{2,3,4,7}$. \\ Pablo Garcia-Pavia ${ }^{1,4,6,8}$
}

(C) Springer Science+Business Media, LLC, part of Springer Nature 2019

Correction to: J. of Cardiovasc. Trans. Res. (2017) 10:35-46 https://doi.org/10.1007/s12265-017-9730-8

The name of author M. Alejandra Restrepo-Cordoba was parsed incorrectly (in such a way as to suggest that her surname is "Alejandra Restrepo-Cordoba") in this article as published.

Please note that "Restrepo-Cordoba" is this author's correct surname.

Publisher's Note Springer Nature remains neutral with regard to jurisdictional claims in published maps and institutional affiliations.

The online version of the original article can be found at https://doi.org/ 10.1007/s12265-017-9730-8

Pablo Garcia-Pavia

pablogpavia@yahoo.es

Fernando Dominguez

fdominguezrodriguez@gmail.com

M. Alejandra Restrepo-Cordoba

ale_res@hotmail.com

Esther Gonzalez-Lopez

esthgonzalez@hotmail.com

Oscar Campuzano

oscar@brugada.org

Tomás Ripoll-Vera

tripol126@gmail.com

Marta Cobo-Marcos

martacobomarcos@hotmail.com

Irene Mademont-Soler

imademont@gencardio.com

Laura Padron-Barthe

lpadron@idiphim.org

Enrique Lara-Pezzi

elara@cnic.es

Luis Alonso-Pulpon

luispulpon@secardiologia.es

Ramon Brugada

ramon@brugada.org

José M. Gámez

jmgamez@hsll.es

Extended author information available on the last page of the article 


\section{Affiliations}

M. Alejandra Restrepo-Cordoba ${ }^{1}$ - Oscar Campuzano ${ }^{2,3,4} \cdot$ Tomás Ripoll-Vera $^{5} \cdot$ Marta Cobo-Marcos $^{1,4}$. Irene Mademont-Soler ${ }^{2,4}$ • José M. Gámez ${ }^{5}$. Fernando Dominguez ${ }^{1,4,6}$ • Esther Gonzalez-Lopez ${ }^{1,4,5}$. Laura Padron-Barthe ${ }^{1,4,6}$ • Enrique Lara-Pezzi ${ }^{4,6}$. Luis Alonso-Pulpon ${ }^{1,4} \cdot$ Ramon Brugada $^{2,3,4,7}$. Pablo Garcia-Pavia ${ }^{1,4,6,8}$

1 Heart Failure and Inherited Cardiac Diseases Unit, Department of Cardiology, Hospital Universitario Puerta de Hierro, Manuel de Falla 2, Majadahonda, 28222 Madrid, Spain

2 Cardiovascular Genetics Centre, Institut d'Investigació Biomèdica de Girona (IDIBGi), Girona, Spain

3 Department of Medical Science, Medical School, Universitat de Girona (UdG), Girona, Spain

4 Centro de Investigacion Biomedica en Red en Enfermedades Cardiovasculares (CIBERCV), Madrid, Spain
5 Department of Cardiology, Hospital Son Llatzer \& IdISPa, Mallorca, Spain

6 Myocardial Biology Programme, Centro Nacional de Investigaciones Cardiovasculares (CNIC), Madrid, Spain

7 Department of Cardiology, Hospital Josep Trueta, Girona, Spain

8 Francisco de Vitoria University, Madrid, Spain 